\title{
Microfluidic enzymatic reactors for proteome research
}

\author{
Yun Liu • Baohong Liu • Pengyuan Yang • \\ Hubert H. Girault
}

Published online: 23 October 2007

(C) Springer-Verlag 2007

\section{Introduction}

The field of proteomics has emerged as a valuable analytical tool for elucidating cellular and biological systems at the molecular level. As there is an ever-growing demand for new highly automated, high-throughput, and sensitive analytical tools, a key challenge is the characterization of lowabundance proteins that are crucial in modulating biological functions of cells and may also be associated with a number of diseases. If the detection modes are mainly dominated by fluorescence spectroscopy and mass spectrometry, the recent advances in microfluidic techniques have the potential to meet the requirements for sample treatment and processing $[1,2]$.

Microfluidic devices can address the future analytical needs of increased throughput, lower sample and reagent consumption, smaller size, and lower operating costs [3]. It has been found that microfluidic-based enzymatic reactors can carry out protein digestion with high efficiency to facilitate subsequent reliable protein identification by peptide mass fingerprinting. Compared to conventional digestion approaches, microfluidic enzymatic reactors can not only accelerate digestion rate but also reduce enzyme autolysis and, ultimately, achieve the purpose of repetitive utilization.

Y. Liu $\cdot$ B. Liu $(\bowtie) \cdot$ P. Yang

Department of Chemistry and Institute of Biomedical Sciences,

Fudan University,

Shanghai 200433, China

e-mail: bhliu@fudan.edu.cn

H. H. Girault

Laboratoire d'Electrochimie Physique et Analytique,

Ecole Polytechnique Fédérale de Lausanne,

Station 6 ,

1015 Lausanne, Switzerland
Fabrication of immobilized microfluidic enzymatic reactors

Typically, microfluidic enzymatic reactors are fabricated by immobilizing enzymes (often trypsin) within the inner surface of a microfluidic channel and on magnetic beads that can be magnetically immobilized in the microchannels. Here, we shall concentrate on the former approach but the methods described for derivatizing microchannels are easily transposable to beads. Three major immobilization mechanisms have been proposed.

1. Covalent binding between the protein and the support matrix: the immobilization takes place in the presence of carbodiimides, cross-linking by glutaraldehyde or cyanogen bromide activation of the support material. The main advantage of covalent binding to the activated support relates to minimization of leakage of the immobilized enzyme. However, inactivation of the enzyme is likely to occur in that many reactive groups of enzyme for immobilization are often located in the active center of the enzyme.

2. Non-covalent binding based on specific physical adsorption between the ligand and the surface of the matrix. Binding forces involve ionic interactions, hydrogen bonds, van der Waals forces, hydrophobic interactions, etc. For example, polyelectrolyte multilayer assemblies have been applied in enzyme adsorption in a controllable manner [4]; this is the simplest way to implement enzyme immobilization on microchips. An advantage of adsorption is that usually no reagents and only a minimum of activation steps are required. However, the leaching of immobilized enzyme tends to occur because of weak non-covalent binding.

3. Sol-gel encapsulation has been used for a decade as a powerful biomolecule encapsulation technique. Forma- 


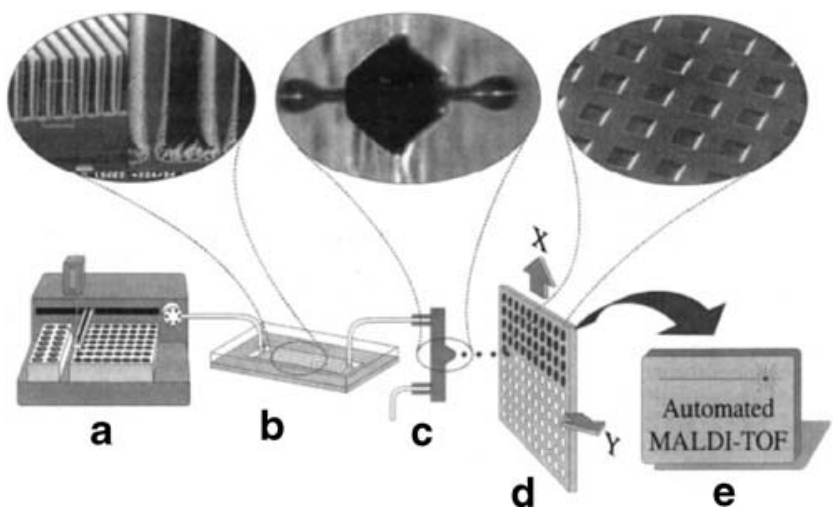

Fig. 1 Microfluidic system for MALDI protein analysis: (a) automated sample pretreatment and injection; (b) m-chip IMER (the photo inset shows a SEM picture of the lamella structure with the porous layer); (c) microdispenser used to deposit sample into m-vials; (d) shallow nanovials $(300 \times 300 \times 20 \mu \mathrm{m})$ on the MALDI target plate; and (e) automated MALDI-TOF-MS analysis. Reprinted from Ref. [10], with permission

tion of a sol-gel begins with the partial or complete hydrolysis of a suitable precursor to form an aqueous solution. After hydrolysis, the sol is used to allow continued evolution of the sol particles. During this time, further hydrolysis and condensation reactions occur, resulting in a wide range of linear, branched and colloidal polysilicates. The hydrolyzed precursor is then mixed with a buffered aqueous solution containing the biomolecules which can be entrapped during the polymerization reaction and gelation [5]. The encapsulation process occurs under mild conditions, and the enzymes retain their native structures and bioactivity for a prolonged period. In recent years, we have developed various sol-gel techniques to modify polymer microfluidic chips for trypsin immobilization, including silica, alumina, and titania sol-gel $[6,7]$.

Several other types of support (for example POROS beads, monolithic chromatographic supports, etc.) are now commercially available or have been specifically developed for immobilization processes $[8,9]$.

\section{Application of microfluidic enzymatic reactors or proteomics}

Most immobilized microfluidic enzymatic reactor applications are currently aimed at protein identification by peptide mapping. Peptide mapping is typically performed using enzymatic cleavage of the protein, and the resulting degraded products are subsequently identified using electrospray ionization-mass spectrometry (ESI MS) or matrix-assisted laser desorption/ionization-mass spectrometry (MALDI MS). Ekstrom et al. [10] described a microchip enzymatic reactor integrated with a sample pretreatment robot and a microdispenser for transfer of digested protein directly to a MALDI target plate for automated MS analysis (Fig. 1). Such a microchip enzymatic reactor allowed online enzymatic digestion of protein samples within 1-3 min, approximately 200-1000 times faster than digestion in solution. Immobilization of the protease has the advantage of avoiding autolytic interferences from the proteolytic enzyme in the mass spectra. By using porous silicon as a carrier for the immobilized enzyme in the microchip reactor, a large surface area and thus a high catalytic turnover are obtained. This integrated system provided a throughput of 100 samples in
Fig. 2 (a) On-chip protocol for protein digestion and identification coupled with MALDI-TOF-MS.

(b) Amplified multilayerassembled enzyme microchip reactor within microchannel. (c) Depiction of layer-by-layer process for enzyme immobilization. Reprinted from Ref. [11], with permission

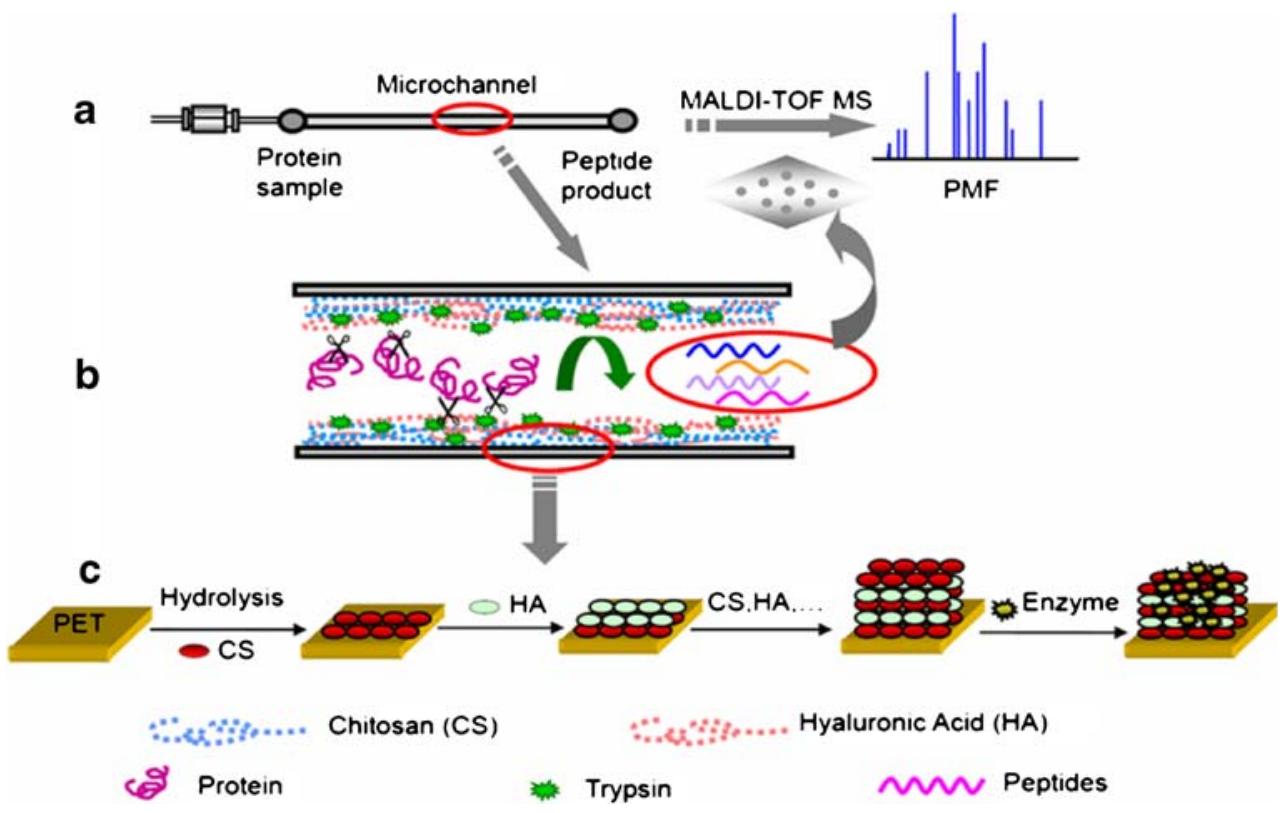


$3.5 \mathrm{~h}$. Figure 2 illustrates the process of enzyme immobilization and subsequent protein identification in a multilayerassembled microchip [11]. Such an assembled membrane provides a biocompatible network with high enzyme-loading capacity. The maximum rate of reaction of the adsorbed trypsin in a microchannel was significantly accelerated. The efficient on-chip proteolysis was obtained within a few seconds and the results of protein identification are comparable with those from in-solution digestion. Both a high surface-to-volume ratio and microstructured confinement within the microchannel induce order-of-magnitude enhancement of catalytic efficiency. Wu et al [12] explored an on-line digestion protocol to couple the microfluidic microreactor integrated with an SST electrode with a replaceable silica-capillary-nanospray tip attached to ESI MS. The experimental data show that the microreactors are suitable for high-throughput analysis of proteins. Numerous micro enzyme-reactors have been developed because they are superior in reducing the reaction time and use minimal amount of reagents. Therefore, these immobilized microfluidic enzyme reactors have also increasingly applied in biosensors [13], and organic or enzymatic synthesis [14-16].

In conclusion, microfluidic enzymatic reactors will become increasingly important to future development and progress of the proteomics field. Due to the high concentration of trypsin that can be confined in the microscopic microchannel, low level of standard protein samples could be rapidly digested in the microchip reactor. One of the advantages of immobilized microfluidic reactors lies in their flexibility, which implies that the approach can be applied in large-scale analysis integrated with separation procedures to implement on-line protein identification.
However, the development of microfluidic enzyme reactors is in the initial stage. It is expected that microfluidic devices, as a burgeoning field, will provide a high-throughput platform for proteomic analysis, imitating biological reaction and biochemical processing.

\section{References}

1. Moorthy J, Burgess R, Yethiraj A, Beebe D (2007) Anal Chem 79:5322-5327

2. Mitchell P (2001) Nat Biotechnol 19:717-721

3. Krenková J, Foret F (2004) Electrophoresis 25:3550-3563

4. Liu Y, Lu HJ, Zhong W, Song PY, Kong JL, Yang PY, Girault HH, Liu BH (2006) Anal Chem 78:801-808

5. Jin W, Brennan J D (2002) Anal Chim Acta 461:1-36

6. Liu Y, Xue Y, Ji J, Chen X, Kong JL, Yang PY, Girault HH, Liu BH (2007) Mol Cell Proteomics 6:1428-1436

7. Liu Y, Qu HY, Xue Y, Wu, Yang PY, Liu BH (2007) Proteomics 7:1373-1378

8. Anderson NL, Haines LR, Pearson TW (2004) J Proteome Res $3: 228-234$

9. Peterson DS, Rohr T, Svec F, Fréchet JMJ (2002) Anal Chem 74:4081-4088

10. Ekstrom S, Onnerfjord P, Nilsson J, Bengtsson M, Laurell T, Marko-Varga G (2000) Anal Chem 72:286-293

11. Liu Y, Zhong W, Meng S, Kong JL, Lu HJ, Yang PY, Girault HH, Liu BH (2006) Chem Eur J 12:6585-6591

12. Wu HL, Zhai JJ, Tian YP, Lu HJ, Wang XY, Jia WT, Liu BH, Yang PY, Xu YM, Wang HH (2004) Lab Chip 4:588-597

13. Xu ZR, Fang ZL (2004) Anal Chim+ Acta 507:129-135

14. Kanno K, Maeda H, Izumo S, Ikuno M, Takeshita K, Tashiro A, Fujii M (2002) Lab Chip 2:15-18

15. Hisamoto H, Shimizu Y, Uchiyama K, Tokeshi M, Kikutani Y, Hibara A, Kitamori T (2003) Anal Chem 75:350-354

16. Srinivasan A, Bach H, Sherman DH, Dordick JS (2004) Biotechnol Bioeng 88:528-535 